\title{
Hallmarks of HIV-1 pathogenesis are modulated by Nef's Secretion Modification Region
}

Kateena Addae Konadu' ${ }^{1}$, Joseph S Anderson ${ }^{2}$, Ming-Bo Huang ${ }^{1}$, Syed A Ali ${ }^{3}$, Michael D Powell ${ }^{1}$, Francois Villinger ${ }^{4}$ and Vincent C Bond ${ }^{1 *}$

${ }^{1}$ Department of Microbiology, Biochemistry and Immunology, Morehouse School of Medicine, Atlanta, Georgia, USA

${ }^{2}$ Department of Internal Medicine, University of California-Davis Medical Center, Sacramento, California, USA

${ }^{3}$ Advanced Medical and Dental Institute, University Sain Malaysia, Pulau Pinang, Malaysia

${ }^{4}$ Department of Pathology and Laboratory Medicine, Emory University School of Medicine and Division of Microbiology and Immunology, Yerkes National Primate Research Center, Atlanta, Georgia, USA

\begin{abstract}
$\mathrm{CD}^{+} \mathrm{T}$ cell depletion and immune activation are hallmarks of HIV infection. Despite extensive studies, the mechanisms underlying immune modulation remain elusive. HIV-1 Nef protein is secreted in exosomes from infected cells and is abundant in the plasma of HIV+ individuals. Exosomal Nef (exNef) was also shown to induce apoptosis in bystander $\mathrm{CD}^{+} \mathrm{T}$ cells. We hypothesized that exNef contributes to HIV pathogenesis. A HIV-1 NL4-3 virus containing alanine substitutions in the secretion modification region (SMR; amino acids 66 to 70; HIVNefsmr5a) was developed. Nef protein containing this modified SMR was shown to be deficient in exNef secretion in nef-transfected cells. Using both HIV-1 NL4-3 wild type (HIVwt) and HIVNefsmr5a, correlates of pathogenesis were evaluated in cell-lines, human peripheral blood mononuclear cells, and humanized NOD-RAG1 $\%$ IL2r $\%$ double mutant (NRG) mice. Disruption of the SMR did not affect viral replication or exNef secretion from infected cell cultures as compared with nef-transfected cells. However, $\mathrm{T}$ cell apoptosis was reduced in HIVNefsmr5a infected cell cultures and $\mathrm{CD} 4^{+} \mathrm{T}$ cell depletion was reduced in the spleen and peripheral blood of similarly infected NRG mice. Inflammatory cytokine release was also decreased in the sera of HIVNefsmr5a infected mice relative to HIVwt infected controls. These findings demonstrate the importance of Nef and the SMR motif in HIV pathogenesis and suggest a potential role for exNef in HIV-driven immune modulation.
\end{abstract}

Keywords: Immune activation; Apoptosis; CD4 T-cells; Exosomes; HIV; Nef; Cytokines

\section{Introduction}

Chronic immune activation and $\mathrm{CD}^{+}$T-cell depletion are hallmarks of HIV infection. Chronic immune activation has been well established as one of the strongest predictors of disease progression [19]. The source of such sustained immune activation has been ascribed to several factors that often use overlapping mechanisms including: (i) continuous plasmacytoid dendritic cell production of Type I IFN [10]; (ii) direct activation via the effect of viral proteins such as Tat, Nef and gp120 [11]; and (iii) bacterial translocation [5]. However, we submit that additional mechanisms underlie immune activation/immune dysregulation. One such candidate is the secretion of exosomes containing the HIV accessory protein Nef.

Nef is a $27-35 \mathrm{kDa}$ protein that is expressed early in the replication cycle of HIV-1 [12]. Nef's myristoylated N-terminal anchor domain is critical for its association with membrane structures and essential for several of its intracellular functions including downregulation of cell surface expression of CD4, MHC-I, and CXCR4 molecules [13]. Several studies have revealed Nef to be a determinant of disease progression in both HIV and SIV infection: (i) Mice and rats transgenic for HIV-1 nef develop immunodeficiencies [14-22]; (ii) the clinical course and both acute and chronic viral loads were markedly attenuated or abolished in both humans and rhesus macaques infected with viral strains that harbor deletions in nef [23-27].

Most of the literature on Nef has centered on its intrinsic functions within infected cells. However, Nef is known to be secreted or released from cells, and has been found in the plasma of HIV positive individuals and SIV infected rhesus macaques [28-29]. Moreover, several investigators, including ourselves, have shown that Nef is excreted from cells and is thereby able to extend its immune regulation beyond infected cells [30-33]. In particular, we have shown that Nef is secreted in small 50-100 $\mathrm{nm}$ diameter vesicular bodies called exosomes characterized by the presence of Alix, AChE, and CD45 [34]. Additionally, exposure of non-infected $\mathrm{CD}^{+} \mathrm{T}$ cells to exosomal $\mathrm{Nef}$ (exNef) resulted in activation induced apoptosis via engagement of the CXCR4 receptor [35]. Genetic analysis revealed several motifs critical for Nef-induced exNef secretion [34]. One previously uncharacterized motif spanning amino acid residues 66-70 (VGFPV), the secretion modification region (SMR), was required for secretion as alanine replacements of the amino acid residues in this motif resulted in abrogation of exNef secretion from nef-transfected cells. This region was also found to be $100 \%$ conserved across HIV-1 clades suggesting the importance of this sequence in a protein highly prone to mutation [34].

Given our findings on the role of exNef and the implication of specific Nef motifs (e.g., SMR) in Nef driven exNef secretion, we examined the role of the SMR motif in Nef-induced pathogenesis. Using HIV1 NL4-3 virus containing alanine substitutions in the SMR developed in our lab (HIVNefsmr5a), we evaluated correlates of in vitro and in vivo pathogenesis in cell lines, human peripheral blood mononuclear cells (PBMCs), and humanized NOD-RAG-1\%IL2r $\%$ double mutant

*Corresponding author: Vincent C Bond, Department of Microbiology, Biochemistry, and Immunology, Morehouse School of Medicine, 720 Westview Drive S.W., Atlanta, GA 30310, USA, Tel: (404) 752-1862, Fax: (404) 752-1179; E-mail: vbond@msm.edu

Received March 04, 2014; Accepted June 19, 2015; Published June 30, 2015

Citation: Konadu KA, Anderson JS, Huang MB, Ali SA, Powell MD, et al. (2015) Hallmarks of HIV-1 pathogenesis are modulated by Nef's Secretion Modification Region. J AIDS Clin Res 6: 476. doi:10.4172/2155-6113.1000476

Copyright: (c) 2015 Konadu KA, et al. This is an open-access article distributed under the terms of the Creative Commons Attribution License, which permits unrestricted use, distribution, and reproduction in any medium, provided the original author and source are credited. 
(NRG) mice. Disruption of the SMR motif in the context of a replication competent HIV isolate did not affect in vitro or in vivo viral replication or exNef secretion from infected cell cultures. This is in sharp contrast with our findings using transfection with plasmids expressing nefsmr $5 a$ where the SMR mutation abrogated exNef secretion [34]. However, $\mathrm{T}$ cell apoptosis was reduced in HIVNefsmr5a infected cell cultures and $\mathrm{CD} 4^{+} \mathrm{T}$ cell depletion was reduced in the spleen and peripheral blood of similarly infected mice. Inflammatory cytokine release was also decreased in the sera of HIVNefsmr5a infected mice relative to HIV wild type (HIVwt) infected controls. These findings suggest that exNef, acting through the SMR motif, may play a significant role in Nef-induced HIV-1 pathogenesis.

\section{Materials and Methods}

\section{HIV-1 plasmid clones (pNL4-3 HIVwt, HIVdsNef, HIVNefsmr5a)}

The HIV-1 pNL4-3 clone was obtained through the NIH AIDS Reagent Program (ARRRP, Figure 1A; 67). To construct the HIVNefsmr5a clone, alanine replacement mutations were generated in pNL4-3 by primer-directed mutagenesis (Figure. 1C) [18]. The HIVdsNef (double stop Nef) mutant of pNL4-3 was generated by direct synthesis of the mutated sequence by Genscript (Piscataway, NJ). The synthetic DNA fragment, containing stop codon mutations (nt 37 and nt 61) and the flanking restriction sites BamHI and XhoI was then ligated into BamHI /XhoI - digested pNL4-3. The resulting dsNef plasmid encodes a Nef reading frame with TGA codons replacing Trp13 and Arg-31 (Figure. 1B) similar to a previously described clone [49].

\section{Production of HIV-1 viral stocks from plasmid clones}

HIV-1 viral stocks were generated by transfection of HEK 293 cells with plasmid DNA. Briefly, HEK 293 cells, grown in RPMI 1640 medium containing $10 \%$ fetal bovine serum, were transfected with $6 \mu \mathrm{g}$ of HIVwt, HIVdsNef, or HIVNefsmr5a NL4-3 viral constructs in T75 flasks by the Effectene transfection reagent (Qiagen, Inc., Valencia, CA). Viral supernatants were harvested $48 \mathrm{~h}$ post transfection and stored at $-80^{\circ} \mathrm{C}$. The concentration of HIV-1 p24 was measured using a p24 capture enzyme-linked immunosorbent assay (ImmunoDiagnostics).

Cell culture. A3.01 CD4 ${ }^{+} \mathrm{T}$ cell lines were maintained at $37^{\circ} \mathrm{C}$ in RPMI 1640 medium supplemented with 10\% heat-inactivated exosome depleted fetal bovine serum, streptomycin $(100 \mathrm{U} / \mathrm{ml})$, penicillin $(100 \mathrm{U} /$ $\mathrm{ml})$, L-glutamine $(2 \mathrm{mM})$, and HEPES-buffered saline solution $(10 \mu \mathrm{M})$. Exosomes were depleted from fetal bovine serum by centrifugation at
$100,000 \times g$ for $16 \mathrm{~h}$ at $4^{\circ} \mathrm{C}$. HEK 293 cells derived from a human primary embryonic kidney transformed with adenovirus type 5, obtained from ARRRP, were maintained at $37^{\circ} \mathrm{C}$ in RPMI 1640 medium supplemented with $10 \%$ heat-inactivated fetal bovine serum, streptomycin $(100 \mathrm{U} / \mathrm{ml})$, penicillin $(100 \mathrm{U} / \mathrm{ml})$, L-glutamine $(2 \mathrm{mM})$, and HEPES-buffered saline solution $(10 \mu \mathrm{M})$. Peripheral blood mononuclear cells (PBMCs) were isolated from HIV seronegative blood donors, obtained from Emory Center for AIDS Research, with Ficoll-Paque. Isolated PBMCs were maintained at $37^{\circ} \mathrm{C}$ in RPMI 1640 medium supplemented with $20 \%$ heat-inactivated exosome depleted fetal bovine serum, streptomycin $(100 \mathrm{U} / \mathrm{ml})$, L-glutamine $(2 \mathrm{mM})$, HEPES-buffered saline solution $(10 \mu \mathrm{M})$, and IL-2 $(20 \mathrm{U} / \mathrm{ml})$. U373-MAGI-CXCR4 $4_{\mathrm{CEM}}$ cells, obtained from the NIH AIDS ARRRP, were maintained at $37^{\circ} \mathrm{C}$ in Dulbecco's modified Eagle's medium supplemented with $10 \%$ heat-inactivated fetal bovine serum, G418 $(0.2 \mathrm{mg} / \mathrm{ml})$, hygromycin B $(0.1 \mathrm{mg} / \mathrm{ml})$, puromycin $(1 \mu \mathrm{g} / \mathrm{ml})$, penicillin $(100 \mathrm{U} / \mathrm{ml})$, and streptomycin $(100 \mathrm{U} /$ $\mathrm{ml}$ ). All participants gave written informed consent, and the study was approved by the institutional review boards of Emory University and Morehouse School of Medicine.

\section{In vitro $\mathrm{HIV}-1$ replication assay}

A3.01 cells maintained at $37^{\circ} \mathrm{C}$ in RPMI 1640 medium supplemented with $10 \%$ heat-inactivated exosome depleted fetal bovine serum, streptomycin $(100 \mathrm{U} / \mathrm{ml})$, penicillin $(100 \mathrm{U} / \mathrm{ml}), \mathrm{L}$-glutamine $(2 \mathrm{mM})$, and HEPES-buffered saline solution $(10 \mu \mathrm{M})$, were exposed to HEK-derived viral supernatants $(25 \mathrm{ng} / \mathrm{p} 24)$ for $24 \mathrm{~h}$. The cells were than pelleted at $1,250 \mathrm{rpm}$ for $10 \mathrm{~min}$ at $25^{\circ} \mathrm{C}$ and re-suspended in fresh media. Viral replication was monitored at 3-day intervals by measuring the amount of p24 present in the culture supernatant. PBMCs maintained at $37^{\circ} \mathrm{C}$ in RPMI 1640 medium supplemented with $20 \%$ heat-inactivated exosome depleted fetal bovine serum, streptomycin $(100 \mathrm{U} / \mathrm{ml})$, L-glutamine $(2 \mathrm{mM})$, HEPES-buffered saline solution $(10 \mu \mathrm{M})$, and IL-2 $(20 \mathrm{U} / \mathrm{ml})$, were exposed to A3.01-derived viral supernatants $(50 \mathrm{ng} / \mathrm{p} 24)$ for $24 \mathrm{~h}$. The cells were than pelleted at $1,250 \mathrm{rpm}$ for $10 \mathrm{~min}$ at $25^{\circ} \mathrm{C}$ and re-suspended in fresh media. Viral replication was monitored at 2-day intervals by measuring the amount of p24 present in the culture supernatant.

\section{Isolation and purification of exosomes from virus}

For the preparation of exosomes on flotation gradients, 40 $\mathrm{ml}$ of supernatants (from uninfected cells or NL4-3 infected cells supernatants) were first centrifuged for $5 \mathrm{~min}$ at $600 \times \mathrm{g}$ to remove cells and cellular debris. The supernatants were then subjected to sequential

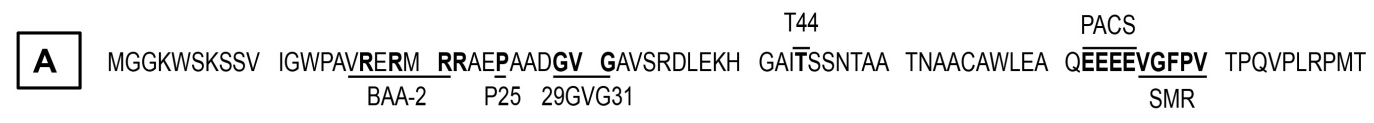

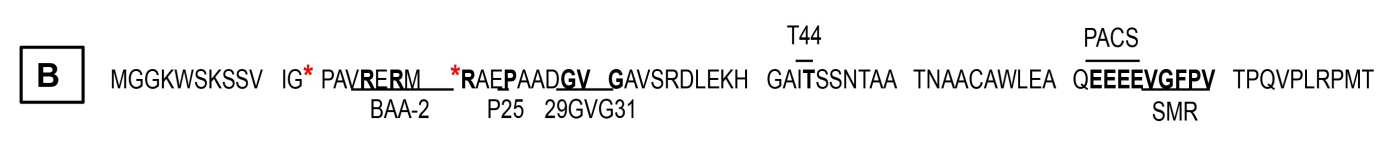

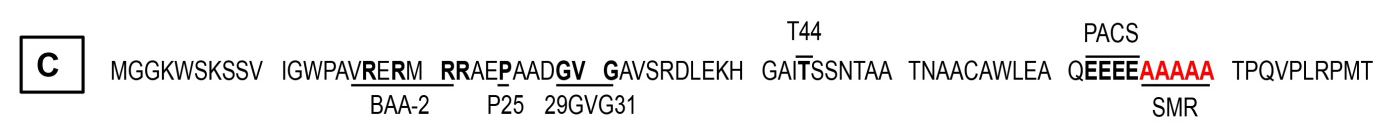

Figure 1: The amino acid sequence of HIV-1 Nef and mutants. (A) HIVwt sequence (1-79aa) showing domains important for Nef-vesicle induced secretion (BAA-2, P25, 29GVG31, T44, PACS motif, and SMR domain). (B) The HIVdsNef mutant contains two point mutations creating two premature termination codons, one 3' of each initiator ATGs. (C) The HIVNefsmr5a mutant contains five alanine substitutions in the Secretion Modification Region (66-70aa). 
centrifugation at $30,000 \times \mathbf{g}$ for $30 \mathrm{~min}$, using a Type FX6100 rotor and at $100,000 \times \mathrm{g}$ for $2 \mathrm{~h}$, using a Type 50.2 ultracentrifuge rotor (Beckman Instruments, Inc., Fullerton, CA). The $100,000 \times \mathbf{g}$ pellets were resuspended in $1 \mathrm{ml}$ of $1 \mathrm{X}$ PBS. Optiprep ${ }^{\mathrm{TM}}$ velocity gradients in PBS were prepared by layering as 11 steps in $1.2 \%$ increments ranging from 6 to $18 \%$. The resuspended $100,000 \times \mathbf{g}$ pellets were layered onto the top of the gradients and centrifuged for $2 \mathrm{~h}$ at $250,000 \times \mathbf{g}$ in a SW40 Ti rotor (Beckman Instruments, Inc.). Gradient fractions containing exosomes (fractions 2-4 = 7.2-9.4\%) were collected, diluted 1:3 with phosphate-buffered saline (PBS), and centrifuged for $2 \mathrm{~h}$ at $400,000 \times$ g using a TLA 100.4 rotor (Beckman Instruments, Inc.). The resultant exosome pellets were used in the TUNEL assay.

\section{MAGI infectivity assay}

Differences in infectivity due to the SMR motif mutation were tested in single round infection of the MAGI cell assay [50]. HIVwt NL4.3, HIVdsNef and HIVNefsmr5a mutant NL4.3 were used to infect A3.01 $\mathrm{CD} 4{ }^{+} \mathrm{T}$ cells. At 12 days post-infection, the supernatants were tested for infection of U373-MAGI-CXCR4 ${ }_{\text {CEM }}$ cells plated in 12-well plates at 1.2 X $10^{5}$ cells/well $24 \mathrm{~h}$ prior to infection. HIV-1 virions $(1 \mathrm{ng} / \mathrm{p} 24)$ were added to each well in duplicate, along with DEAE-dextran at a final concentration of $20 \mu \mathrm{g} / \mathrm{ml}$. Forty-eight hours post-infection, cells were fixed by incubation with $0.2 \%$ glutaraldehyde and $1 \%$ formaldehyde for $5 \mathrm{~min}$ at room temperature. Following fixation, cells were washed with $\mathrm{PBS}$ and stained for $60 \mathrm{~min}$ at $37^{\circ} \mathrm{C}$ with $\mathrm{X}-\mathrm{Gal}$ solution (5-bromo-4chloro-3-indolyl- $\beta$-D-galactosidase [Invitrogen] dissolved in dimethyl sulfoxide, $4 \mathrm{mM}$ potassium ferrocyanide, $4 \mathrm{mM}$ ferricyanide, and 2 $\mathrm{mM} \mathrm{MgCl}$ in PBS). The cells were washed twice with PBS, and positive syncytia (blue-stained cells) were counted with an inverted microscope (model TMS-F; Nikon, Melville, NY). Results are reported as the total number of blue cells per ng of p24.

\section{TUNEL assay}

Jurkat $\mathrm{CD} 4^{+} \mathrm{T}$ cells were assayed for apoptosis using a TUNEL assay as described previously [35]. The cells were visualized by epifluorescence on a computer-controlled microscope system (Zeiss microscope; Carl Zeiss, Thornwood, NY). Microscopic images were processed using a charge-couple device camera, MC 100 SPOT (Photonic Science, East Sussex, United Kingdom). Further image processing was conducted with the Image-Pro Plus 2.0 software (Media Cybernetics, Silver Spring, MD).

\section{Immunoblot analysis}

Cells and exosome proteins were analyzed using Western blot analysis. The protein samples were separated by SDS-PAGE on $4-20 \%$ Tris-HCL Criterion precast gel (Bio-Rad Laboratories, Hercules, CA) and electrophoretically transferred to a nitrocellulose membrane. The membrane was washed in Tris-buffered saline (TBS; Bio-Rad) for $5 \mathrm{~min}$, blocked with $5 \%$ nonfat milk in TTBS (TBS with $0.1 \%$ Tween 20) for $1 \mathrm{~h}$ by shaking at room temperature, and processed for immunoblotting using a specific primary antibody with shaking at $4^{\circ} \mathrm{C}$ overnight, followed by a secondary HRP-conjugated IgG $(\mathrm{H}+\mathrm{L})$ antibody. Protein bands were detected by using the Western blotting luminol reagent (Santa Cruz Biotechnology) and imaged with the Image Quant LAS 4000 (GE Healthcare Biosciences, Pittsburgh, PA). Images were arranged for publication in Adobe illustrator 10 (Adobe Systems, San Jose, CA). The following antibodies were used: (i) murine monoclonal anti-Nef antibody (Immuno Diagnostics, Inc., Bedford, MA); (ii) murine monoclonal anti-p24 antibody (Immuno Diagnostics, Inc.); (iii) goat anti-mouse IgG $(\mathrm{H}+\mathrm{L})$ labeled with horseradish peroxidase (HRP) antibody (Thermo Fisher Scientific, Inc., Rockford, IL).

\section{Flow cytometry analysis}

To evaluate the effect that mutation of the SMR motif had on CD4 and CXCR4 downregulation, A3.01 CD4 ${ }^{+} \mathrm{T}$ cells were stained with antihuman antibodies and analyzed by flow cytometry. Cells were stained with allophycocyanin (APC)/cyanine 7 (CY7)-labeled anti-CD4 (OKT4; Biolegend, San Diego, CA) and phycoerythrin (PE)/CY7-labeled antiCXCR4 (12G5; Biolegend). Viability was assessed with Alexa Fluor 430-Carboxylic Acid, Succinimidyl Ester (Life Technologies, Grand Island NY). To evaluate the percentage of $\mathrm{CD}^{+} / \mathrm{CD}^{+} \mathrm{T}$ cells in infected PBMC cultures, T cells were stained with Alexa Fluor 700-labeled antiCD3 (UCHT1; BD Bioscience), APC/CY7-labeled anti-CD4 (OKT4; Biolegend). Viability was assessed with Alexa Fluor 430 Carboxylic Acid, Succinimidyl Ester (Invitrogen). Flow cytometry analysis was performed on a $\mathrm{BD}^{\mathrm{TM}}$ LSR II (BD Bioscience) cytometer. Flow cytometry data was acquired with the Diva software and analyzed using FlowJo software 10.0.5 (Tree Star, Inc, Ashland, OR).

\section{Humanized mouse studies}

The humanized mouse model used is described in detail in Walker et al., 2012 [51]. Briefly, CD34 ${ }^{+}$hematopoietic stem cells (HSCs) were obtained from umbilical cord blood obtained after normal delivery (NDRI, Philadelphia, PA) using Ficoll-Paque (GE Healthcare, Piscataway, NJ). HSCs were purified by immunomagnetic bead separation (Miltenyi Biotec, Auburn, CA). A CD34+ cell purity of $>91 \%$ was routinely obtained. NOD-RAG1-/-IL2r $\gamma$-/- double mutant (NRG) mice (stock number 007799) were obtained from The Jackson Laboratory (Sacramento, CA). Mice were used in compliance with institutional and IACUC guidelines. Newborn NRG pups (2-5 days old) were sublethally irradiated with $150 \mathrm{cGy}$ of gamma irradiation and subsequently intrahepatically injected with $3.5 \times 10^{5}$ cells/mouse. Three months post-transplant, mice were bled and the peripheral blood was analyzed by flow cytometry for human leukocytes with PE-CY7-labeled anti-CD45 (HI30; BD Bioscience), APC-labeled anti-CD3 (HIT3A; BD Bioscience), and PE-labeled anti-CD4 (RPA-T4; BD Bioscience). Flow cytometry was performed on a Beckman Coulter FC-500.

\section{HIV-1 infection of engrafted NRG mice}

Successfully engrafted NRG mice were infected intra-peritoneally with 200,000 TCID $_{50}$ of HIV-1 (either NL4.3 HIVwt or NL4.3 HIVNefsmr5a). The infected mice were monitored for HIV replication in vivo by measuring human $\mathrm{CD} 4^{+}$cells in blood and plasma viremia every two weeks. Peripheral blood was analyzed for total human $\mathrm{CD} 4^{+}$ cell percent by flow cytometry with PE-CY7-labeled anti-CD45 (HI30; BD Bioscience), APC-labeled anti-CD3 (HIT3A; BD Bioscience), and PE-labeled anti-CD4 (RPA-T4; BD Biosciences). Flow cytometry was performed on a Beckman Coulter FC-500.

To determine levels of HIV plasma viremia, viral RNA was extracted from the blood of infected mice using a Qiagen Viral RNA extraction kit (Qiagen, Valencia, CA). Reverse transcription was performed with oligo dT primers using a Taqman RT Reagents kit (Applied Biosystems, Carlsbad, CA). Quantitative-PCR was performed using SYBR Green (Applied Biosystems, Carlsbad, CA) and a probe and primer pair specific for the HIV pol gene: 5'-CTGGCTACTATTTCTTTTGCTA-3' and 5'-TGGCATGGGTACCAGCACA-3' and probe 5'-TTTATCTACTTGTTCATTTCCTCCATTCCTT-3' (IDT DNA Technologies, Coralville, IA). Quantitative-PCR was performed on an Applied Biosystems 7200 analyzer. 


\section{Luminex multiplex assay}

Twenty cytokines/chemokines were measured in plasma from HIV1 infected and uninfected NRG mice using a high-sensitivity human cytokine/chemokine 20-plex magnetic bead kit (Affymetrix, Inc, Santa Clara, CA) comprising CD40 ligand, GM-CSF, IFN- $\alpha 2$, IFN- $\beta$, IFN- $\gamma$, IL-1 $\beta$, IL-6, IL-7, IL-8, IL-10, IL-12p70, IL-13, IL-15, IP-10, MCP-1, MIP-1 $\alpha$, MIP-1 $\beta$, TNF- $\alpha$, Fas ligand and ICAM-1. Data was acquired using a luminex-200 system and analyzed using Bio-plex Manager software, 6.0 (Bio-Rad).

\section{Data analysis}

The numerical and graphical analyses of all in vitro data were based on at least three repetitions of each experiment. Statistical analysis was performed and graphs were generated using either SigmaPlot 10 (Systat, San Jose, CA) or GraphPad Prism 6.0 (GraphPad Software, Inc., La Jolla, CA). Statistical significance was evaluated with an unpaired two-factor $t$ test set at a $p$ level of $<0.05$. On account of the relative small sample size, NRG mice data was log-transformed prior to analysis.

\section{Results}

\section{Mutation of the SMR motif results in delayed viral release and decreased infectivity}

We have previously identified motifs in Nef that are required for Nef-induced exNef secretion [34]. One of those motifs, the secretion modification region, is conserved across all HIV-1 clades. This motif was found to be important for secretion as alanine replacements of its five amino acids completely abolished exNef secretion in nef-transfected cells [34]. Based on the reports suggesting a role for exNef in Nefinduced pathogenesis, we sought to examine the effect of the mutation of the SMR motif on correlates of pathogenesis. To evaluate the effect of the SMR motif on HIV replication and infectivity, we constructed a replication competent HIV-1 NL4-3 clone expressing a nef gene with five alanine replacements in the SMR motif (HIVNefsmr5a; Figure. 1C). As controls, we used the wild type HIV-1 NL4-3 clone (HIVwt; Figure $1 \mathrm{~A}$ ), and a clone expressing a nef gene with point mutations resulting in two premature stop codons (HIVdsNef; Figure 1B). A3.01 $T$ cells were infected with viral supernatants corresponding to the same amount of p24 prepared from HEK 293 cells transfected with each of these viral clones. The cells were cultured for 12 days and monitored at 3-day intervals for viral release by p24 ELISA and for infectivity by MAGI assay. While the levels of p24 released from HIVwt infected A3.01 cells started to increase by day 3 , peaking by day 7, there was a slight delay in p24 release from cells infected with HIVNefsmr5a as well as HIVdsNef, with peak p24 release observed at day 9 (Figure $2 \mathrm{~A}$ ). To examine the effect of the SMR mutation on infectivity, culture supernatants harvested from infected A3.01 cells were assessed for infectivity by MAGI assay. HIVNefsmr5a viral particles were found to be 10 -fold less infectious than HIVwt viral particles, while HIVdsNef viral particles were 100-fold less infectious (Figure 2B).

SMR motif is necessary for CXCR4 downregulation: Among its numerous intrinsic functions, Nef is known to downregulate the cell surface expression of HIV co-receptors CD4, CXCR4, and major histocompatibility complex (MHC) class I [13]. The reduction in the expression of these molecules is thought to prevent super-infection, facilitate viral release from infected cells, and assist in immune evasion [13]. These functions of Nef have been mapped to structural motifs within the protein. The motifs for CD4 downregulation are located at the N-terminal arm and the C-terminal loop, specifically amino acid residues W57 and L58 [36-38], and a dileucine motif L164-L165 [39-
A

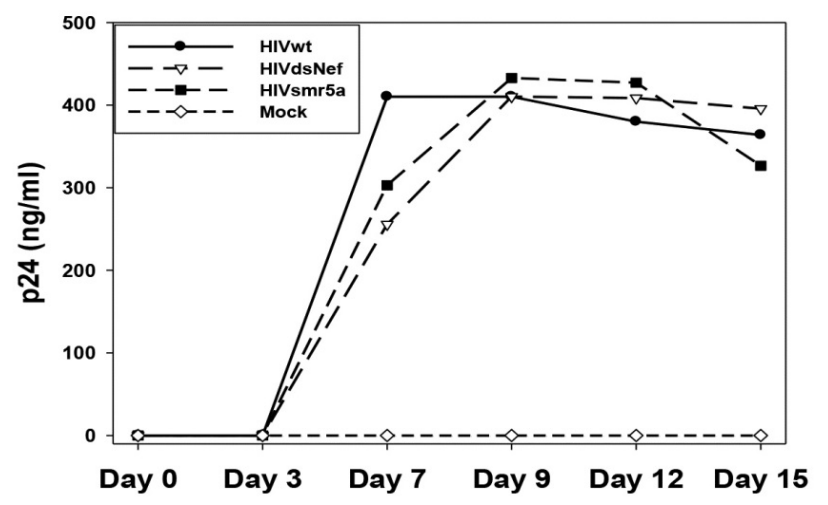

B

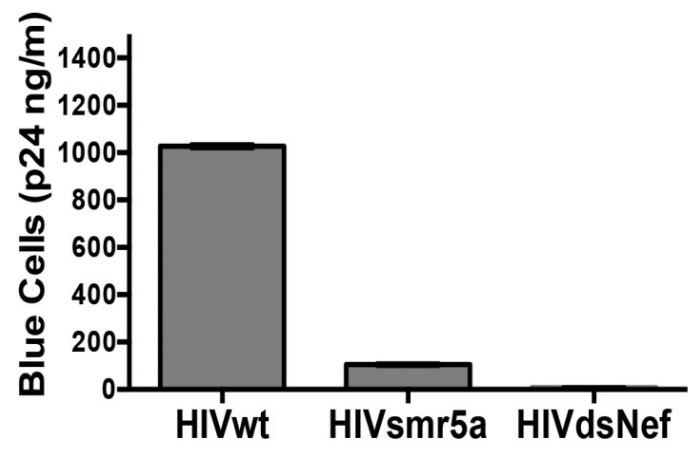

Figure 2: Mutation of the SMR motif results in delayed viral release and decreased infectivity. (A) $5.0 \times 10^{5} \mathrm{~A} 3.01 \mathrm{~T}$ cells were infected with HIVwt, HIVdsNef, or HIVNefsmr5a virus and assayed by ELISA for changes in p24 release in culture medium at 3-day intervals. Differences in viral replication kinetics were observed between the various viral clones. (B) A3.01-derived viral preparations were assessed for infectivity by MAGI assay. HIVNefsmr5a and HIVdsNef viral particles were found to be less infectious than HIVwt viral particles by 10 -fold and 100 -fold respectively. Plots shown are a representation of three independent experiments.

41]. Nef-mediated downregulation of CXCR4 and MHC-I are located in the phosphofurin acidic cluster sequence (PACS) and the Src homology domain 3 (SH3) motifs [42-43]. The SMR motif is located between the $\mathrm{PACS}$ and SH3 motifs, which prompted us to investigate the impact of mutation of the SMR motif on Nef-mediated down regulation of CD4 and CXCR4. Aliquots of the A3.01 T cell line were infected with either HIVNefsmr5a, HIVwt, or HIVdsNef and subsequently analyzed for surface expression of CD4 and CXCR4 by flow cytometry. While mutation of the SMR motif only modestly affected CD4 downregulation relative to HIVwt, it abolished the ability of Nef to downregulate CXCR4 (Figure 3B and 3C). To rule out the possibility that decreased expression of Nef within HIVNefsmr5a infected cells contributed to this observation, similar amounts of cell lysates, measured by total protein, from infected cultures were assessed for expression levels of Nef by western blot analysis. Nef was found to be expressed in equal amounts in HIVNefsmr5a infected cultures as compared to HIVwt infected cultures (Figure 3A).

SMR motif is dispensable for viral replication in human PBMCs: To extend the analyses of the role of the SMR in primary cell cultures, 
A

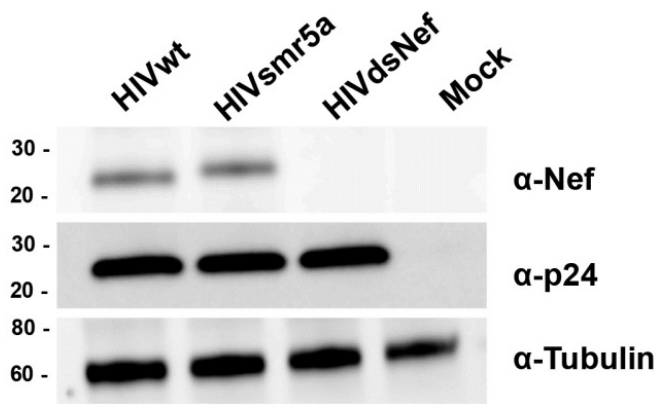

B
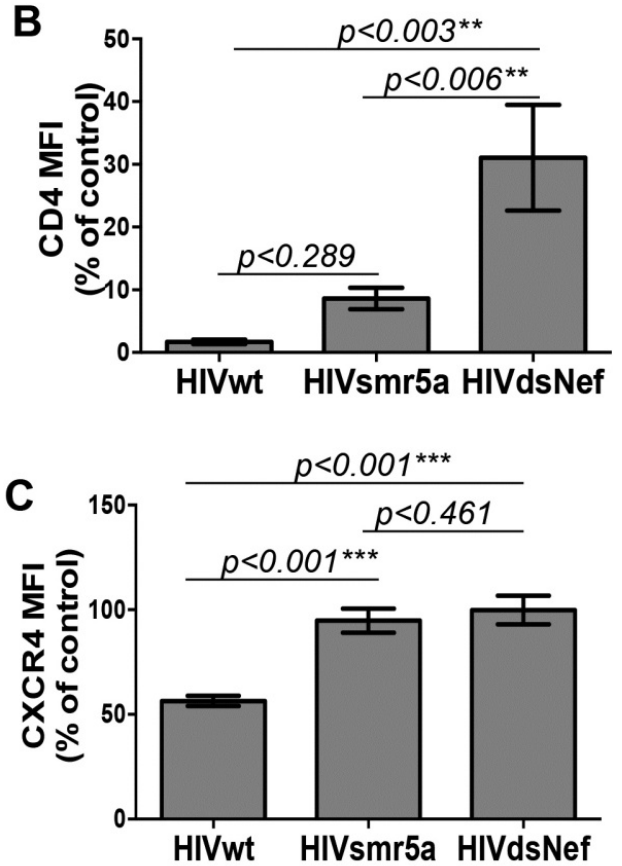

Figure 3: SMR motif is necessary for CXCR4 downregulation. (A) Cell lysates of A3.01 T cells infected with each viral clone were analyzed 12 days post-infection for Nef expression level. Nef was found to be expressed in equal amounts in HIVNefsmr5a infected cultures as compared to HIVwt infected cultures. Image shown is a representative image from three independent experiments. (B-C) A3.01 T cells infected with HIVNefsmr5a, HIVwt, and HIVdsNef were analyzed for surface expression of CD4 and CXCR4 by flow cytometry. Mutation of the SMR motif abolished the ability of Nef to downregulate CXCR4. Nef-mediated downregulation of CD4 was slightly affected. Values reflect the means of three independent experiments, and error bars represent SEM of the mean. Differences between groups were determined using an unpaired student $t$-test ${ }^{*} p<0.05,{ }^{* *} p<0.01,{ }^{* * *} p<0.001$.

viral replication was compared in primary cultures of human PBMCs. Isolated PBMCs were infected with equal amounts, based on p24 content, of HIVNefsmr5a, HIVdsNef, or HIVwt viral supernatants. The cells were cultured for 12 days and monitored at 3-day intervals for viral release by p24 ELISA. Unlike the infection of the A3.01 cell line, p24 release from HIVNefsmr5a- and HIVwt-infected PBMCs displayed similar viral replication kinetics for donors 1 and 2, while viral release was delayed in HIVdsNef-infected cultures (Figure 4A). Conversely, donor 3 displayed similar kinetics as observed using the A3.01 infected cell line (Figure 4A).

\section{$\mathrm{CD}^{+} \mathrm{T}$ cell depletion was reduced in HIVNefsmr5a infected PBMC cultures}

To determine whether infection with HIVNefsmr5a virus resulted in depletion of $\mathrm{CD} 4^{+} \mathrm{T}$ cells in culture, isolated PBMCs were infected with equal amounts of HIVNefsmr5a, HIVdsNef, or HIVwt viral supernatants. The cells were cultured for 12 days, harvested, and examined for viable levels of $\mathrm{CD}^{+} / \mathrm{CD}^{+} \mathrm{T}$ cells by flow cytometry. Compared to PBMC cultures infected with HIVwt in which only $27.8 \%$ of $\mathrm{CD} 4^{+} / \mathrm{CD}^{+} \mathrm{T}$ cells remained at 12 days post infection, HIVNefsmr5a-infected cultures exhibited $51.2 \%$ of $\mathrm{CD}^{+} / \mathrm{CD}^{+}$live $\mathrm{T}$ cells and HIVdsNef cultures $63.6 \%$ (Fig. 4B). The difference between HIVNefsmr5a and HIVwt cultures was statistically significant $(p<0.027)$, while the difference observed between HIVNefsmr5a and HIVdsNef was not $(p<0.231)$. However, compared to mock infected cultures (79.9\%), a significant difference was observed in HIVwt- $(p<0.001)$ and HIVNefsmr5a- $(p<0.021)$ cultures, while no significant difference was observed in HIVdsNefcultures ( $\mathrm{p}<0.116$; Fig. 4B).

\section{ExNef is released from HIVwt and HIVNefsmr5a infected} cells

Nef is known to be released in exosomes from HIV-infected and $n e f$-transfected cells [30-33]. In previous studies from our group, an important SMR motif for nef-induced exNef secretion was identified using a nef-transfection assay [34]. To examine the effect of mutation of the SMR motif on exNef release during the course of a viral infection, PBMC were infected with HIVNefsmr5a virus and culture supernatants were assayed for exNef. To separate exosomes from HIV-1 particles, conditioned cell-free media, 12 days post-infection, was harvested and concentrated by ultracentrifugation at $30,000 \times \mathbf{g}$ for $30 \mathrm{~min}$ and 100,000 $\times \mathbf{g}$ for $2 \mathrm{~h}$, re-suspended in $1 \mathrm{X}$ PBS then separated by centrifugation through a $6-18 \%$ Optiprep ${ }^{\mathrm{TM}}$ velocity separation gradient. Fractions from each gradient were collected and assayed for $\mathrm{AChE}$, an exosomal marker, by enzymatic assay, and p24 to confirm purification of exosomes from viral particles. Fractions were also assayed by Western blot analysis, probing for Nef and p24. Using AChE and p24 to identify exosomes and virus respectively, exosomes sediment in fractions 2-4 (7.2-9.4\%) (Fig. 5B), while HIV-1 viral particles sediment in fractions 8-10 (14.4-16.8\%; Fig. 5A). Contrary to our previous finding in neftransfected cells, Nef was detected in both the exosomes and the viral particles collected from cells infected with HIVwt and HIVNefsmr5a (Fig. 5C).

\section{ExNef from HIVNefsmr5a infected cells results in reduced apoptosis}

We and others have shown that exNef induces apoptosis in HIV naïve and uninfected $\mathrm{CD} 4^{+} \mathrm{T}$ cells [31-35]. Given the finding that 


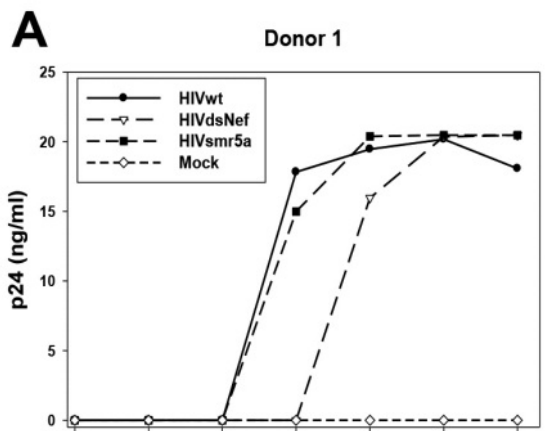

Day 0 Day 2 Day 4 Day 6 Day 8 Day 10 Day 12

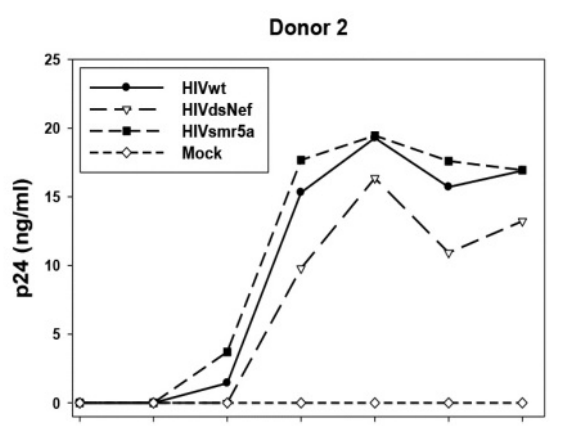

Day 0 Day 2 Day 4 Day 6 Day 8 Day 10 Day 12

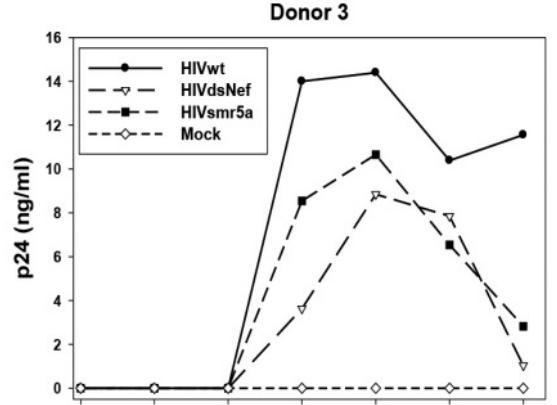

Day 0 Day 2 Day 4 Day 6 Day 8 Day 10 Day 12

B

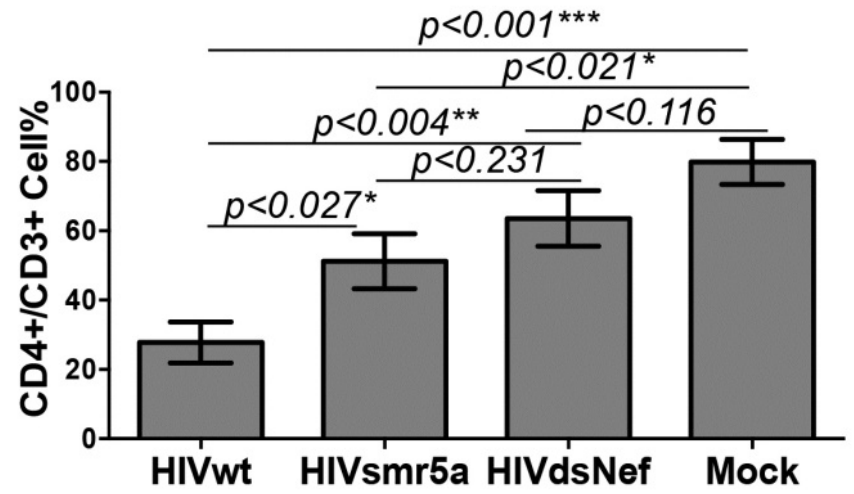

Figure 4: SMR is dispensable for viral replication and CD4+/CD3+ T cell depletion was reduced in HIVNefsmr5a infected PBMCs. (A) 4.0 X10^6 PBMCs from three HIV-1 seronegative donors were infected with HIVwt, HIVdsNef, or HIVsmr5A virus and assayed by ELISA for changes in p24 release in culture medium at 2-day intervals. p24 ELISA analysis revealed similar viral replication kinetics for HIVwt and HIVNefsmr5a viral clones for donors 1 and 2 . (B) $4.0 \times 10^{\wedge} 6$ PBMCs from nine HIV-1 seronegative donors were infected with HIVwt, HIVdsNef, or HIVsmr5A virus. Day 12 post infection, cells were harvested and analyzed for the percentage of $\mathrm{CD}^{+}$lymphocytes expressing CD4 by flow cytometry. The percentage of CD3 ${ }^{+}$lymphocytes expressing CD4 was significantly higher in HIVNefsmr5a infected cultures relative to HIVwt. Values reflect the means of three independent experiments with three different donors used per experiment. Error bars represent SEM of the mean. Differences between groups were determined using an unpaired student $t$-test ${ }^{*} p<0.05,{ }^{* *} p<0.01,{ }^{* * *} p<0.001$.

exNef was released in the culture supernatant of HIVNefsmr5a infected cells, we next investigated whether exNef from HIVNefsmr5a infected cells were able to induce apoptosis in HIV naïve cells similar to exNef released from HIVwt infected cells. To remain in the physiological range in which Nef exosomes are found in the plasma of HIV positive individuals $(10 \mathrm{ng} / \mathrm{ml})$ [28], Jurkat cells were exposed to $30 \mathrm{ng} / \mathrm{ml}$ of exosomes isolated from HIVNefsmr5a or HIVwt infected PBMC cultures for $48 \mathrm{~h}$. The cultures were subsequently screened for apoptosis by TUNEL assay. Compared to exNef from HIVwt (7.5\%) infected cells, there was significant reduction in the number of cells undergoing apoptosis exposed to exNef from HIVNefsmr5a (2.6\%) infected cells $(p<0.036$; Figure 5D) which was essentially similar to that seen in Jurkat cells exposed to exosomes isolated from mock infected cultures $(2.2 \%$, $p<0.768$; Figure 5D).

\section{$\mathrm{CD4}^{+} \mathrm{T}$ cell depletion was reduced in HIVNefsmr5a-infected NRG mice}

Although effects of SMR mutations were observed in vitro, it was unclear whether mutation of the SMR motif would have any consequences in vivo. This issue prompted us to use the humanized NRG mouse model. We examined the effect of infection with HIVNefsmr5a or HIVwt on viral loads, human $\mathrm{CD}^{+} \mathrm{T}$ cell dynamics and cytokine/ chemokine responses using this model. Surprisingly, the difference in viral dynamics between HIVwt and HIVNefsmr5a-infected NRG mice were minimal and similar to the kinetics observed in in vitro infected PBMC cultures from donors 1 and 2 (Fig. 6A and 4A). In fact, the levels of HIV-1 RNA copies per milliliter were between 7 and 8 log units in HIVNefsmr5a-infected mice at 9 weeks post-infection, which was equivalent to the levels of HIV-1 RNA copies per milliliter in HIVwtinfected controls.

In contrast to plasma viremia, significant differences were observed for human $\mathrm{CD}^{+} \mathrm{T}$ cell dynamics in mice infected with HIVwt compared to those inoculated with HIVNefsmr5a (Figure. 6B, C, and D). The peripheral blood at various weeks post infection was monitored for levels of $\mathrm{CD}^{+} \mathrm{T}$ cells using standard flow cytometry. While mice infected with HIVwt displayed a significant, gradual decline (Figure. 6B), in the frequencies of human $\mathrm{CD} 4^{+} \mathrm{T}$ cells (as determined on the gated population of human $\mathrm{CD} 45^{+}$leukocytes) from an average of $79.45 \%$ pre-infection to an average of $26.2 \%$ at 9 weeks post-infection $(p<0.05$; Figure. $6 \mathrm{C}$ ), mice infected with HIVNefsmr5a maintained relatively stable or slightly increasing frequencies of human $\mathrm{CD}^{+} \mathrm{T}$ cell levels throughout the 9 weeks of follow-up (Figure. 6B), from $54.26 \%$ preinfection to $68.7 \%$ at 9 weeks post-infection (Figure. $6 \mathrm{C}$ ). To verify that the $\mathrm{CD} 4^{+} \mathrm{T}$ cell levels measured in blood were not a reflection of altered trafficking patterns, the levels of human $\mathrm{CD} 4^{+} \mathrm{T}$ cells were evaluated in the spleen of infected mice at the end of the challenge experiments. 
A

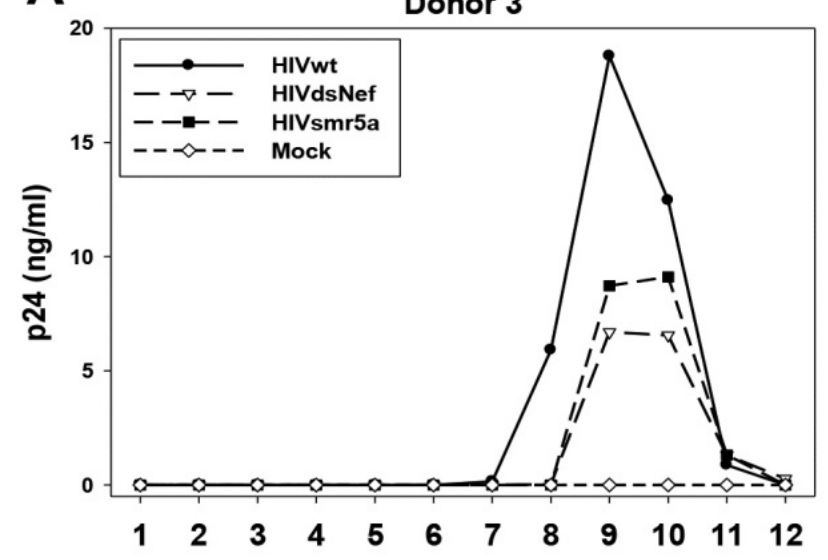

C

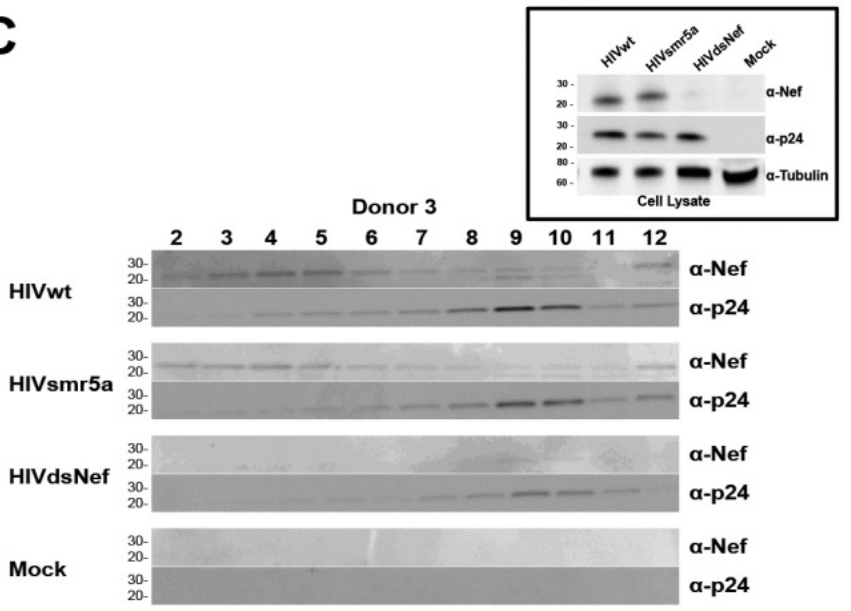

B

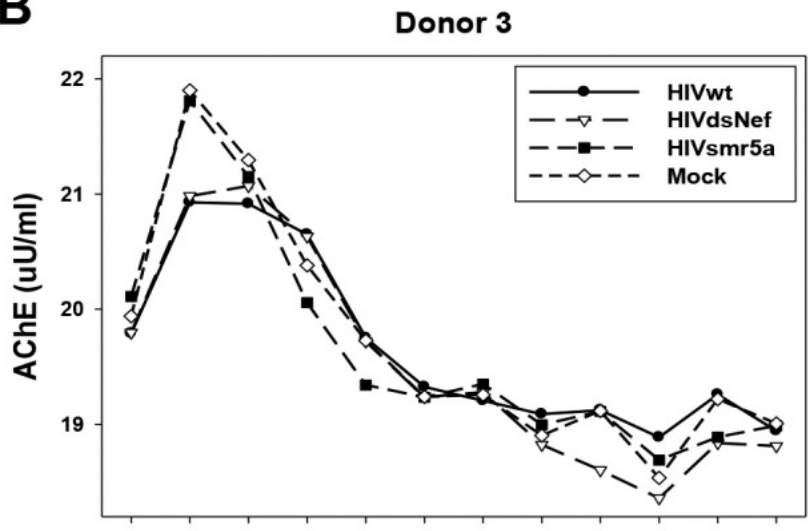

$\begin{array}{lllllllllllll}1 & 2 & 3 & 4 & 6 & 6 & 7 & 8 & 9 & 10 & 11 & 12\end{array}$

D

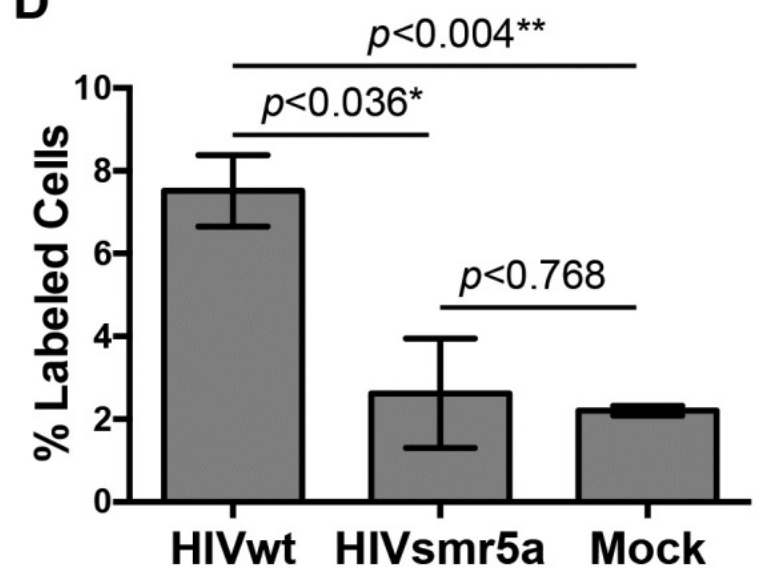

Figure 5: ExNef is released from HIVNefsmr5a infected cells. (A) $4.0 \times 10^{6} \mathrm{HIV}$ seronegative PBMCs were infected with HIVwt, HIVdsNef, or HIVNefsmr5a virus. 12 days post-infection, conditioned cell-free media was harvested and subjected to differential centrifugation at $30,000 \times g$ for $30 \mathrm{~min}$ and $100,000 \times g$ for $2 \mathrm{~h}$. The 100,000 $\times g$ pellet was re-suspended in $1 \mathrm{ml}$ of $1 \mathrm{X}$ PBS, loaded onto Optiprep ${ }^{\mathrm{TM}}$ velocity separation gradient, and subjected to flotation centrifugation at $250,000 \times g$ for $2 \mathrm{~h}$. (A-C) Fractions were assayed by p24 and AChE enzymatic assay, a marker for exosomes, and western analysis for Nef and p24 to confirm purification of exosomes from viral particles. While Nef was not detected in the exosome, or the viral fractions of supernatants collected from cells infected with HIVdsNef, Nef was detected in both the exosome, and the viral fractions of supernatants collected from cells infected with HIVwt and HIVNefsmr5a. Plots shown are a representative image from one donor of three independent experiments (D) Exosomes isolated from donor \#3 was screened for apoptosis by TUNEL assay. Exosomes were added to target cell cultures at $30 \mathrm{ng} / \mathrm{ml}$ as measured by total protein. Compared to exNef from HIVwt infected cells, there was a significant reduction in the number of cells undergoing apoptosis after $48 \mathrm{~h}$ of exposure to exNef from HIVNefsmr5a infected cells. Error bars represent SEM from the mean. Differences between groups were determined using an unpaired student $t$-test ${ }^{*} p<0.05,{ }^{* *} p<0.01$.

There was a significant decrease $(p<0.05)$ in the percentage of human $\mathrm{CD} 4^{+} \mathrm{T}$ cells in the spleen of HIVwt infected mice $(31.5 \%)$ compared to HIVNefsmr5a infected mice (70.8\%; Figure. 6D), indicating that the decrease in $\mathrm{CD}^{+} \mathrm{T}$ cells observed in blood was generalized.

Pro-inflammatory cytokine levels were elevated in HIVwtinfected NRG mice but not in HIVNefsmr5a-infected NRG mice.

The discrepancy between viral loads and CD4 loss suggested a difference in pathogenic mechanisms. We therefore investigated whether mutation of the SMR motif resulted in difference in the elicitation of pro-inflammatory cytokines in HIVNefsmr5a- versus HIVwt-infected mice during acute and chronic infection. Plasma specimens were collected from mice at 2 and 9 weeks post-infection, and analyzed by multiplex Luminex array for a panel of 20 human cytokines/chemokines (Supplementary Table 1). Among these 20 analytes at week 2, IL-13 $(p<0.001)$ and IL-15 $(p<0.01)$ were significantly elevated in HIVwt-infected mice but not in HIVNefsmr5ainfected mice relative to uninfected controls (Figure. 7A). At week 9 (Figure. 7B), IL-7 ( $p<0.001)$, IL-10 ( $p<0.01)$, IL-15 ( $p<0.001)$, and IP-10 $(p<0.001)$ were significantly elevated in HIVwt-infected mice but not in HIVNefsmr5a-infected mice. In fact, HIVNefsmr5a- infected mice did not show significant elevation for any of the tested analytes at the specified time points relative to uninfected controls.

\section{Discussion}

Nef is known to be secreted from HIV-infected and nef-transfected cells, though the exact role of extracellular Nef in HIV pathogenesis remains to be fully elucidated [14-17]. One report in the nonhuman primate model of HIV showed that secreted SIV Nef was able to 
A

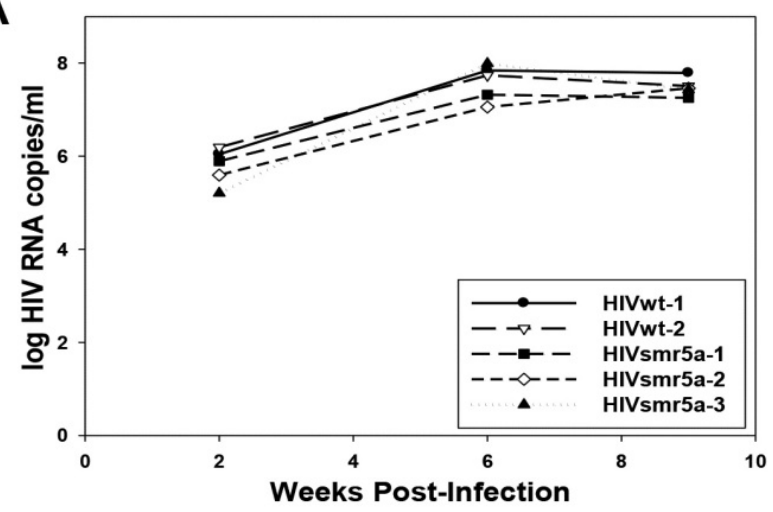

C

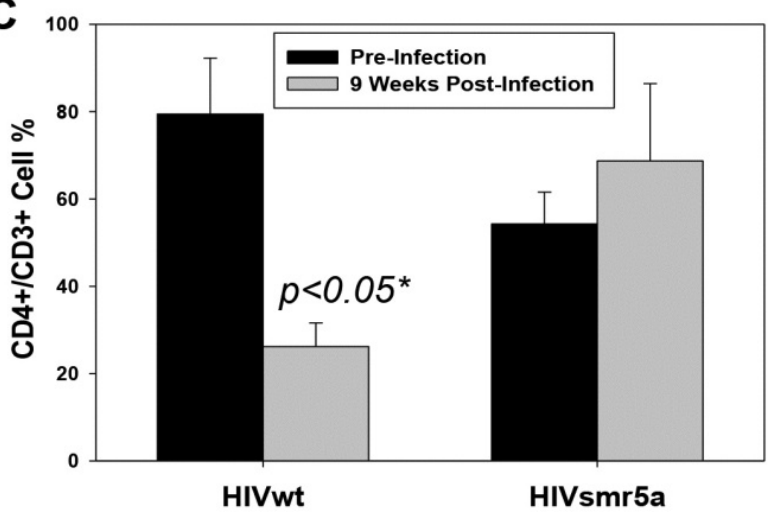

B 100

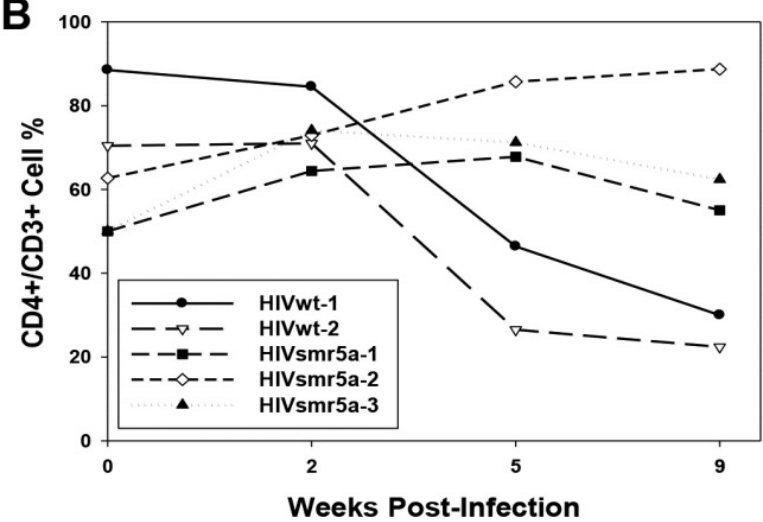

D

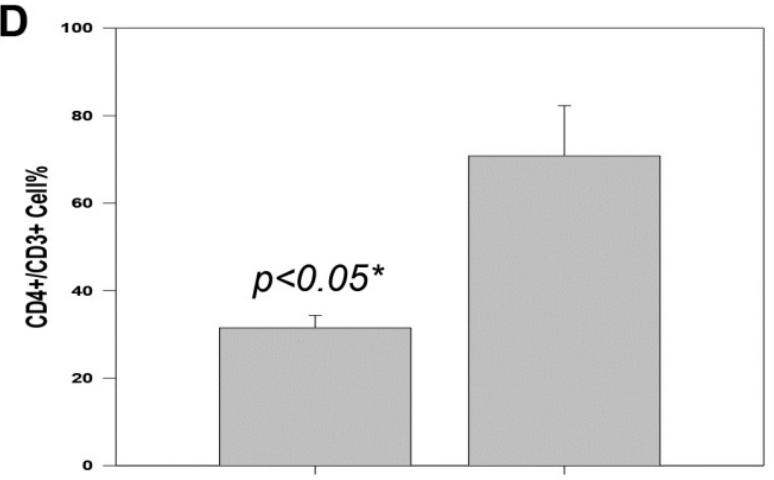

HIVwt

HIVsmr5a

Figure 6: $\mathrm{CD}^{+} / \mathrm{CD}^{+} \mathrm{T}$ cell depletion was reduced in HIVNefsmr5a infected NRG mice. NRG mice successfully transplanted with CD34 ${ }^{+} \mathrm{HSC}$ s were infected with either HIVwt $(n=2)$ or HIVNefsmr5a $(n=3)$ NL4-3 virus. (A-C) At various weeks post-infection, mice were bled and the plasma was analyzed by Q-PCR using a primer/ probe pair specific for HIV pol gene and PBMCs were analyzed by flow cytometry for total CD45 human $C D 4^{+} / C D 3^{+} \mathrm{T}$ cell percentage. (A) Disruption of the SMR motif did not impair in vivo viral replication and (B-C) disruption of the SMR motif resulted in a significant reduction in depletion of CD4 $4^{+}$CD $3^{+}$T cells relative to HIVwt. (D) Disruption of the SMR motif also resulted in preservation of $\mathrm{CD} 4^{+} / \mathrm{CD} 3^{+} \mathrm{T}$ cells in the spleen of HIVNefsmr5a infected mice relative to HIVwt. Error bars represent SEM from the mean. Differences between groups were determined using an unpaired student $t$-test $\quad{ }^{*} p<0.05$.

A
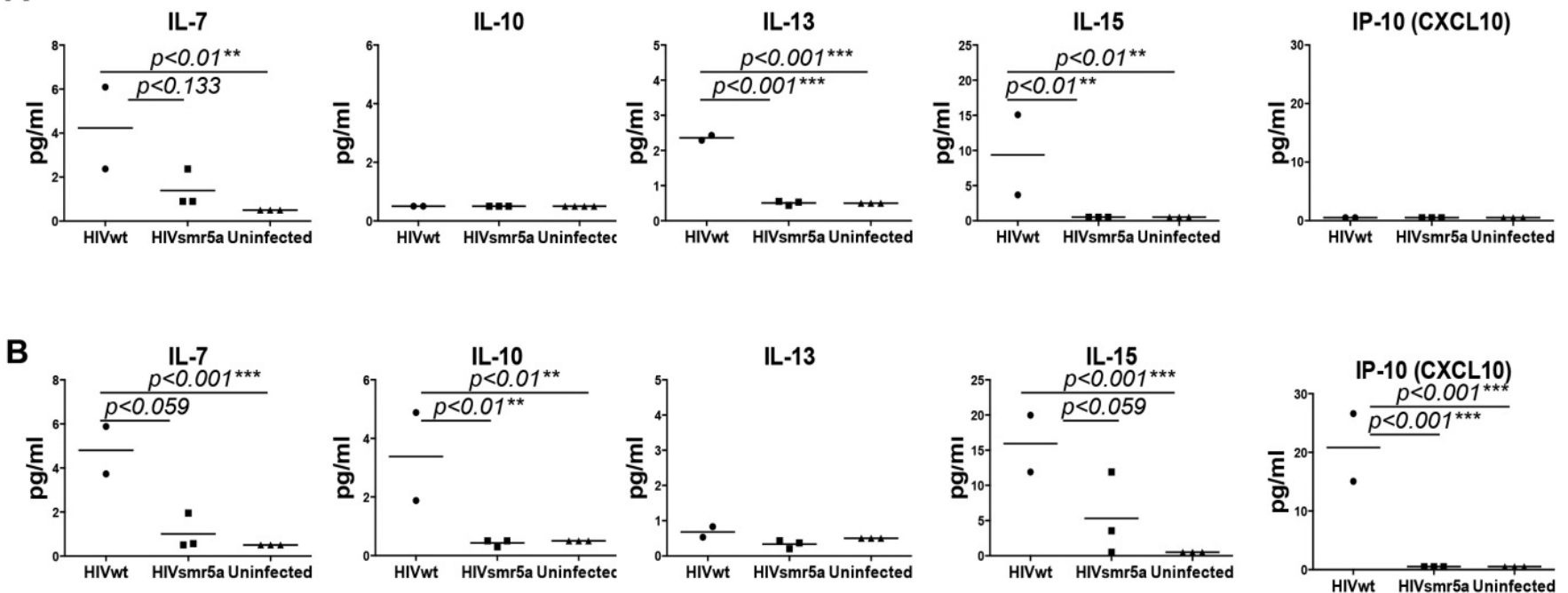

Figure 7: Pro-inflammatory cytokine levels were reduced in HIVNefsmr5a infected NRG mice. NRG mice successfully transplanted with CD34+ HSCs were infected with either HIVwt $(n=2)$ or HIVNefsmr5a $(n=3)$ NL4-3 virus. At various weeks post-infection, mice were bled and the plasma was analyzed for pro-inflammatory cytokine and chemokine expression by 20 -plex luminex array. Of the 20 pro-inflammatory cytokines and chemokines measured, (A) Week 2 post-infection: IL-13, and IL-15 were significantly increased in HIVwt infected mice relative to HIVNefsmr5a and (B) Week 9 post-infection: IL-10 and IP-10 was significantly elevated in HIVwt infected mice compared to HINefsmr5a. Differences between groups were determined using an unpaired student $t$-test ${ }^{\star} p<0.05$, ${ }^{* *} p<0.01$, ${ }^{* * *} p<0.001$. 
affect hematopoiesis [29], although the authors did not test whether Nef was or was not released in exosomes. Importantly, the secretory function was mapped to specific motifs near the N-terminus of the Nef protein. One of these motifs, the previously uncharacterized secretion modification region, was found to be conserved across all HIV-1 clades [34]. However, despite this observation, little is know about this region and its involvement in exNef secretion and Nef-induced pathogenesis.

In this study, we investigated the role of the SMR motif in correlates of pathogenesis during the course of an HIV infection using both in vitro and in vivo model systems. Alanine replacements of the five amino acid residues of the SMR did not attenuate viral replication in A3.01 T cells, similar results were seen in Jurkat $\mathrm{T}$ cells (data not shown), but instead resulted in delayed viral release compared to HIVwt control with virus eventually reaching levels similar to those in the control. Conversely, mutation of this region did affect Nef-mediated enhancement of infectivity. In fact, infectivity of HIVNefsmr5a viral particles was 10fold less infectious than HIVwt. This observation may explain the delay in viral release from HIVNefsmr5a-infected A3.01 T cells suggesting this region maybe important for post-entry steps in HIV-1 target cells.

One surprising finding in our study was the release of Nef in exosomes from HIVNefsmr5a infected cells. In our previous analysis [34], mutation of the SMR in NL4-3 nef cloned into expression vector pQBI resulted in abolition of exNef secretion in nef-transfected cell. But as shown here, mutation of this region in the context of the replicative did not result in abrogation of exNef secretion. This finding suggests that in the presence of the full-length virus, other compensatory mechanisms may rescue exNef release, further underscoring the importance of this pathway for the virus.

While Nef was produced and released in exosomes from HIVNefsmr5a-infected cells, exNef from these cells induced far less apoptosis in bystander cells, than exNef from HIVwt infections. Interestingly, this functional phenotype was mapped to two apoptotic motifs, one of which (Nef amino acids 50 to 60 ) is located five amino acids upstream of the SMR motif [44]. The The second motif was mapped to amino acids 170 to 180 . Recently, we showed by molecular modeling that mutation of the SMR region of Nef affected the threedimensional structure of the Nef protein [45]. Thus, we reason that the change of residues 66-70 (VGFPV to AAAAA) in the HIVNefsmr5a mutant would most likely alter the function of the adjacent apoptotic motif (Nef amino acid residues 50-60). In fact, intracellular and extracellular Nef within and released from HIVNefsmr5a appeared to have a higher molecular weight than HIVwt upon separation by SDSPAGE. This observation was true for both human T cell lines and human primary cells (data not shown). Taken together, our findings suggest that mutation of the SMR region could have altered the presentation of the apoptotic motif in exNef to target cells thereby resulting in a reduction of Nef-induced apoptosis.

One well established intrinsic function of Nef is to downregulate the cell surface expression of the HIV co-receptors CD4 and CXCR4, and the major histocompatibility complex MHC-I. Since SMR is located between the motifs responsible for Nef-mediated down regulation of CXCR4 and MHC-I, PACS and SH3, we investigated the effect of mutation of this region on cell surface expression of these molecules in infected cells. Similar to Meuwissen et al., the HIVNefsmr5a mutant failed to completely down regulate CXCR4 [46]. Conversely, Nefmediated down regulation of $\mathrm{CD} 4$ was not affected. Our results, in line with Meuwissen and coworkers, indicate the SMR is essential for CXCR4 down regulation, but is not required for Nef-mediated CD4 down regulation [46].
As a final set of in vitro studies, we investigated whether the SMR motif was necessary for viral replication in human PBMC. In our assay, the SMR motif was dispensable for viral replication as HIVNefsmr5a virus replicated as efficiently as the HIVwt, while there was a slight delay in viral release from HIVdsNef-infected cells. In contrast, Meuwissen et al. reported a significant attenuation in replicative ability of virus containing a mutation in the SMR region (VGF to AAA) and premature stop codons in Nef [46]. Since the same proviral backbone was used in our study, we speculate that the difference stems from the culture methods for infecting primary cells. Our PBMC cultures were not activated prior to infection and cultures were infected with a larger concentration of virus. This may explain the discrepancy between our results and their findings. In fact, infecting cell cultures with virus stocks greater than 300ng of p24 resulted in matched replication kinetics for HIVwt, HIVNefsmr5a, and HIVdsNef (data not shown).

One salient finding in the infection of primary human PBMCs in vitro and in vivo with HIVNefsmr5a was while its replication kinetics matched HIVwt, HIVNefsmr5a appeared to spare CD4+ T cells (Figure. $4 \mathrm{~B}$ and 6 ). While the reasons for this dichotomy are unclear, one may posit this is a reflection of a direct effect exerted in infected cells. This indeed may be true, but would indicate markedly altered intrinsic Nef functions since HIVNefsmr5a appeared to produce intracellular levels of Nef equivalent to HIVwt (Figure. 3A and 5C). However, our data also clearly points to differences in apoptosis induction capacity of exNef released from HIVNefsmr5a- versus HIVwt-infected cell cultures (Figure. 5D). It is possible that mutation of the SMR altered the structure of Nef both intracellularly and in exosomes leading to functional alterations both intracellularly and extracellularly. Indeed, mutation of the SMR resulted in the loss of the virus ability to downregulate CXCR4 (Figure. 4C). In addition, mutation of the SMR could have also affected the three-dimensional structure of Nef leading to alteration in the function of the adjacent apoptotic motif shown to be responsible for the exNef apoptotic effect [44]. Taken together, this may help in explaining in vitro and in vivo preservation of $\mathrm{CD} 4^{+} \mathrm{T}$ cells during infection with HIVNefsmr5a despite matched viral replication kinetics with HIVwt.

The alteration of Nef function was also reflected in the absence of detectable immune activation in mice infected with HIVNefsmr5a, relative to HIVwt- and mock-infected mice. Serum levels of proinflammatory cytokines IL-7, IL-10, IL-13, IL-15, and IP-10, which were significantly elevated in HIVwt-infected mice, were found to be at levels similar to uninfected controls in mice Infected with HIVNefsmr5a. Interestingly, IL-7 and IL-15 were shown to be elevated in plasma from women with acute HIV-1 infection [47]. Further, IL-7 was found to be associated with rapid CD4 loss [47], likely reflecting compensatory secretion. In the same study, elevated levels of IP-10 was significantly associated with higher HIV viral loads, and levels of IL-10 correlated directly with acute infection viral loads and a greater risk of $\mathrm{CD} 4^{+} \mathrm{T}$ cell loss. Collectively, our findings suggest the SMR motif may influence the pro-inflammatory cytokine storm observed during acute infection, which is thought to contribute to the rate of disease progression in HIV infection.

In a study conducted by Stoddart et al. mutations of the regions involved in CD4 down regulation, amino acid residues 57/58 and $164 / 165$, resulted in attenuation of pathogenesis in severe combined immunodeficiency mice implanted with human fetal thymus and liver (SCID-hu Thy/Liv mice) [48]. Alternatively, SCID-hu Thy/liv mice infected with virus containing a mutation in the PACS region displayed pathogenic potential comparable to that of wild type virus 
Citation: Konadu KA, Anderson JS, Huang MB, Ali SA, Powell MD, et al. (2015) Hallmarks of HIV-1 pathogenesis are modulated by Nef's Secretion Modification Region. J AIDS Clin Res 6: 476. doi:10.4172/2155-6113.1000476

[48]. These findings demonstrated that a loss in the ability of the virus to down regulate cell surface expression of MHC-I is dispensable for pathogenesis, while down regulation of CD4 is required for Nefmediated pathogenesis. In our study, mutation of the SMR did not affect the ability of the virus to down regulate CD4. It is therefore interesting to speculate whether this virus would exhibit an attenuated phenotype in a model of human HIV pathogenesis. The reduced levels of proinflammatory cytokines and preservation of $\mathrm{CD} 4^{+} \mathrm{T}$ cells certainly argues in favor of attenuation, though further confirmation is required given the limited sample size in our in vivo study. Overall, our in vitro and in vivo findings suggest that the SMR motif may play an important role in HIV-1 pathogenesis.

\section{Acknowledgements}

We thank the following persons: Jane Chu, Michelle Lang, William Roth, Alexander Quarshie, Eduardo Lani Volpe Da Silveira, Kenneth A. Rogers, Martin N. Shelton, Mafuz Khan, Chunxia Zhao, Richard Barnard, Praveen K. Amancha, Wendy Armstrong, Cameron Tran, and Emory University Center for AIDS Research. Kateena Addae Konadu was supported by UNCF/Merck Graduate Research Dissertation Fellowship, American Medical Association Foundation, CRECD Grant 8R25MD007589-10, and NIH NIGMS MBRS Grant R25 GM058268. This work was supported by Grant Numbers 8G12MD007602 and 8U54MD007588 from NIMHD, Georgia Research Alliance grant GRA.VAC08.W, and Emory CFAR grant P30 A1050409. The following reagents were obtained through the NIH AIDS Research and Reference Reagent Program, Division of AIDS, NIAID, NIH: U373MAGI-CXCR4CEM cells. The authors declare they have no conflicts of interest.

\section{References}

1. Levacher M, Hulstaert F, Tallet S, Ullery S, Pocidalo JJ, et al. (1992) The significance of activation markers on CD8 lymphocytes in human immunodeficiency syndrome: staging and prognostic value. Clin Exp Immunol 90: 376-382

2. Giorgi JV, Liu Z, Hultin LE, Cumberland WG, Hennessey K, et al. (1993) Elevated levels of CD38+ CD8+ T cells in HIV infection add to the prognostic value of low CD4+ T cell levels: results of 6 years of follow-up. J Acq Immun Def Synd 6: 904-912.

3. Bofill M, Mocroft A, Lipman M, Medina E, Borthwick NJ, et al. (1996) Increased numbers of primed activated $C D 8+C D 38+C D 45 R O+T$ cells predict the decline of CD4+ T cells in HIV-1-infected patients. AIDS 10: 827-834.

4. Liu Z, Cumberland WG, Hultin LE, Prince HE, Detels R, et al. (1997) Elevated CD38 antigen expression on CD8+ T cells is a stronger marker for the risk of chronic HIV disease progression to AIDS and death in the Multicenter AIDS Cohort Study than CD4+ cell count, soluble immune activation markers, or combinations of HLA-DR and CD38 expression. J Acquir Immune Defic Syndr Hum Retrovirol 16: 83-92

5. Douek DC, Roederer M, Koup RA (2009) Emerging concepts in the immunopathogenesis of AIDS. Annu Rev Med 60: 471-484.

6. Roberts L, Passmore JA, Williamson C, Little F, Bebell LM, et al. (2010) Plasma cytokine levels during acute HIV-1 infection predict HIV disease progression. AIDS 24: 819-831.

7. Mueller YM, Petrovas C, Bojczuk PM, Dimitriou ID, Beer B, et al. (2005) Interleukin-15 increases effector memory CD8+ t cells and NK Cells in simian immunodeficiency virus-infected macaques. J Virol 79: 4877-4885.

8. Picker LJ, Reed-Inderbitzin EF, Hagen SI, Edgar JB, Hansen SG, et al. (2006) IL-15 induces CD4 effector memory T cell production and tissue emigration in nonhuman primates. J Clin Invest 116: 1514-1524.

9. Mueller YM, Do DH, Altork SR, Artlett CM, Gracely EJ, et al. (2008) IL-15 treatment during acute simian immunodeficiency virus (SIV) infection increases viral set point and accelerates disease progression despite the induction of stronger SIV-specific CD8+ T cell responses. J Immunol 180: 350-360.

10. O'Brien M, Manches O, Bhardwaj N (2013) Plasmacytoid dendritic cells in HIV infection. Adv Exp Med Biol 762: 71-107.

11. Chihara T, Hashimoto M, Osman A, Hiyoshi-Yoshidomi Y, Suzu I, et al. (2012) HIV-1 proteins preferentially activate anti-inflammatory M2-type macrophages. J Immunol 188: 3620-3627.

12. Kim SY, Byrn R, Groopman J, Baltimore D (1989) Temporal aspects of DNA and RNA synthesis during human immunodeficiency virus infection: evidence for differential gene expression. J Virol 63: 3708-3713.
13. Landi A, lannucci V, Nuffel AV, Meuwissen P, Verhasselt B (2011) One protein to rule them all: modulation of cell surface receptors and molecules by HIV Nef. Curr HIV Res 9: 496-504.

14. Dickie $P$ (1996) HIV type 1 Nef perturbs eye lens development in transgenic mice. AIDS Res Hum Retroviruses 12: 177-189.

15. Dickie P (2000) Nef modulation of HIV type 1 gene expression and cytopathicity in tissues of HIV transgenic mice. AIDS Res Hum Retroviruses 16: 777-790.

16. Dickie P, Ramsdell F, Notkins AL, Venkatesan S (1993) Spontaneous and inducible epidermal hyperplasia in transgenic mice expressing HIV-1 Nef. Virology 197: 431-438.

17. Hanna Z, Kay DG, Rebai N, Guimond A, Jothy S, et al. (1998) Nef harbors a major determinant of pathogenicity for an AIDS-like disease induced by HIV-1 in transgenic mice. Cell 5: 163-175.

18. Hanna Z, Weng X, Kay DG, Poudrier J, Lowell C, et al. (2001) The pathogenicity of human immunodeficiency virus (HIV) type 1 Nef in CD4C/HIV transgenic mice is abolished by mutation of its $\mathrm{SH} 3$-binding domain, and disease development is delayed in the absence of Hck. J Virol 75: 9378-9392.

19. Kajiyama W, Kopp JB, Marinos NJ, Klotman PE, Dickie P (2000) Glomerulosclerosis and viral gene expression in HIV-transgenic mice: role of nef. Kidney Int 58: 1148-1159.

20. Kay DG, Yue P, Hanna Z, Jothy S, Tremblay E, et al. (2002) Cardiac disease in transgenic mice expressing human immunodeficiency virus-1 nef in cells of the immune system. Am J Pathol 161: 321-335.

21. Simard MC, Chrobak P, Kay DG, Hanna Z, Jothy S, et al. (2002) Expression of simian immunodeficiency virus nef in immune cells of transgenic mice leads to a severe AIDS-like disease. J Virol 76: 3981-3995.

22. Skowronski J, Parks D, Mariani R (1993) Altered T cell activation and development in transgenic mice expressing the HIV-1 nef gene. EMBO $\mathrm{J} 12$ 703-713.

23. Deacon NJ, Tsykin A, Solomon A, Smith K, Ludford-Menting M, et al. (1995) Genomic structure of an attenuated quasi species of HIV-1 from a blood transfusion donor and recipients. Science 270: 988-991.

24. Kestler HW 3rd, Ringler DJ, Mori K, Panicali DL, Sehgal PK, et al. (1991) Importance of the nef gene for maintenance of high virus loads and for development of AIDS. Cell 65: 651-662.

25. Kirchhoff F, Greenough TC, Brettler DB, Sullivan JL, Desrosiers RC (1995) Brief report: absence of intact nef sequences in a long-term survivor with nonprogressive HIV-1 infection. N Engl J Med 332: 228-232.

26. Learmont JC, Geczy AF, Mills J, Ashton LJ, Raynes-Greenow CH, et al. (1999) Immunologic and virologic status after 14 to 18 years of infection with an attenuated strain of HIV-1. A report from the Sydney Blood Bank Cohort. N Engl J Med 340: 1715-1722.

27. Salvi R, Garbuglia AR, Di Caro A, Pulciani S, Montella F, et al. (1998) Grossly defective nef gene sequences in a human immunodeficiency virus type 1-seropositive long-term nonprogressor. J Virol 72: 3646-3657.

28. Fujii $Y$, Otake K, Tashiro M, Adachi A (1996) Soluble Nef antigen of HIV-1 is cytotoxic for human CD4+ T cells. FEBS Lett 393: 93-96.

29. Prost S, Le Dantec M, Augé S, Le Grand R, Derdouch S, et al. (2008) Human and simian immunodeficiency viruses deregulate early hematopoiesis through a Nef/PPARgamma/STAT5 signaling pathway in macaques. J Clin Invest 118 : 1765-1775.

30. Campbell TD, Khan M, Huang MB, Bond VC, Powell MD (2008) HIV-1 Nef protein is secreted into vesicles that can fuse with target cells and virions. Ethn Dis 18: S2-14-9.

31. Muratori C, Cavallin LE, Krätzel K, Tinari A, De Milito A, et al. (2009) Massive secretion by $T$ cells is caused by HIV Nef in infected cells and by Nef transfer to bystander cells. Cell Host Microbe 6: 218-230.

32. Lenassi M, Cagney G, Liao M, Vaupotic T, Bartholomeeusen K, et al. (2010) HIV Nef is secreted in exosomes and triggers apoptosis in bystander CD4+ T cells. Traffic 11: 110-122.

33. Raymond AD, Campbell-Sims TC, Khan M, Lang M, Huang MB, et al. (2011) HIV Type 1 Nef is released from infected cells in CD45(+) microvesicles and is present in the plasma of HIV-infected individuals. AIDS Res Hum Retroviruses 27: $167-178$ 
Citation: Konadu KA, Anderson JS, Huang MB, Ali SA, Powell MD, et al. (2015) Hallmarks of HIV-1 pathogenesis are modulated by Nef's Secretion Modification Region. J AIDS Clin Res 6: 476. doi:10.4172/2155-6113.1000476

34. Ali SA, Huang MB, Campbell PE, Roth WW, Campbell T, et al. (2010) Genetic characterization of HIV type 1 Nef-induced vesicle secretion. AIDS Res Hum Retroviruses 26: 173-192.

35. James CO, Huang MB, Khan M, Barrio MG, Powell MD, et al. (2004) Extracellular Nef protein tragets CD4+ T cells for apoptosis by interacting with CXCR4 surface receptors. J Virol 78: 3009-3109.

36. Grzesiek S, Stahl SJ, Wingfield PT, Bax A (1996) The CD4 determinant for downregulation by HIV-1 Nef directly binds to Nef. Mapping of the Nef binding surface by NMR. Biochemistry 35: 10256-10261.

37. Lu X, Yu H, Liu SH, Brodsky FM, Peterlin BM (1998) Interactions between HIV1 Nef and vacuolar ATPase facilitate the internalization of CD4. Immunity 8: 647656.

38. Mangasarian A, Piguet V, Wang JK, Chen YL, Trono D (1999) Nef-induced CD4 and major histocompatibility complex class I (MHC class I) down-regulation are governed by distinct determinants: $\mathrm{N}$-terminal alpha helix and proline repeat of Nef selectively regulate MHC class I trafficking. J Virol 73: 1964-1973.

39. Bresnahan PA, Yonemoto W, Ferrell S, Williams-Herman D, Geleziunas R, et al. (1998) A dileucine motif in HIV-1 Nef acts as an internalization signal for CD4 downregulation and binds the AP-1 clathrin adaptor. Curr Biol 8: 1235-1238.

40. Greenberg M, DeTulleo L, Rapoport I, Skowronski J, Kirchhausen T (1998) A dileucine motif in HIV-1 Nef is essential for sorting into clathrin-coated pits and for downregulation of CD4. Curr Biol 8: 1239-1242.

41. Lock M, Greenberg ME, lafrate AJ, Swigut T, Muench J, et al. (1999) Two elements target SIV Nef to the AP-2 clathrin adaptor complex, but only one is required for the induction of CD4 endocytosis. EMBO J 18: 2722-2733.

42. Geyer M, Fackler OT, Peterlin BM (2001) Structure--function relationships in HIV-1 Nef. EMBO Rep 2: 580-585.
43. Foster JL, Denial SJ, Temple BR, Garcia JV (2011) Mechanisms of HIV-1 Nef function and intracellular signaling. J Neuroimmune Pharmacol 6: 230-246.

44. Huang MB, Jin LL, James CO, Khan M, Powell MD, et al. (2004) Characterization of Nef-CXCR4 interactions important for apoptosis induction. J Virol 78: 1108411096.

45. Campbell PE, Isayev O, Ali SA, Roth WW, Huang MB, et al. (2012) Validation of a novel secretion modification region (SMR) of HIV-1 Nef using cohort sequence analysis and molecular modeling. J Mol Model 18: 4603-4613.

46. Meuwissen PJ, Stolp B, lannucci V, Vermeire J, Naessens E, et al. (2012) Identification of a highly conserved valine-glycine-phenylalanine amino acid triplet required for HIV-1 Nef function. Retrovirology 9: 34.

47. Roberts L, Passmore JA, Williamson C, Little F, Bebell LM, et al. (2010) Plasma cytokine levels during acute HIV-1 infection predict HIV disease progression. AIDS 24: 819-831.

48. Stoddart CA, Geleziunas R, Ferrell S, Linquist-Stepps V, Moreno ME, et al (2003) Human immunodeficiency virus type 1 Nef-mediated downregulation of CD4 correlates with Nef enhancement of viral pathogenesis. J Virol 77: 21242133.

49. Chowers MY, Spina CA, Kwoh TJ, Fitch NJ, Richman DD, et al. (1994) Optimal infectivity in vitro of human immunodeficiency virus type 1 requires an intact nef gene. J Virol 68: 2906-2914.

50. Khan M, Garica-Barrio M, Powell MD (2001) Restoration of wild-type infectivity to human immunodeficiency virus type 1 strains lacking nef by intravirion reverse transcription. J Virol 75: 12081-12087.

51. Walker JE, Chen RX, McGee J, Nacey C, Pollard RB, et al. (2012) Generation of an HIV-1-resistant immune system with CD34(+) hematopoietic stem cells transduced with a triple-combination anti-HIV lentiviral vector. J Virol 86: 57195729 . 